\title{
A GENERALIZATION OF CERTAIN CLASS OF ANALYTIC FUNCTIONS WITH NEGATIVE COEFFICIENTS
}

\author{
M. K. AOUF AND A. SHAMANDY
}

\begin{abstract}
We introduce the subclass $T^{*}(A, B, n, \alpha) \quad(-1 \leq A<B \leq 1,0<$ $B \leq 1, n \geq 0$, and $0 \leq \alpha<1$ ) of analytic functions with negative coefficients by the operator $D^{n}$. Coefficient estimates, distortion theorems, closure theorems and radii of close-to-convexety, starlikeness and convexity for the class $T^{*}(A, B, n, \alpha)$ are determined. We also prove results involving the modified Hadamard product of two functions associated with the class $T^{*}(A, B, n, \alpha)$. Also we obtain several interesting distortion theorems for certain fractional operators of functions in the class $T^{*}(A, B, n, \alpha)$. Also we obtain class perserving integral operator of the form
\end{abstract}

$$
F(z)=\frac{c+1}{z^{c}} \int_{0}^{z} t^{c-1} f(t) d t, \quad c>-1
$$

for the class $T^{*}(A, B, n, \alpha)$. Conversely when $F(z) \in T^{*}(A, B, n, \alpha)$, radius of univalence of $f(z)$ defined by the above equation is obtained.

\section{Introduction}

let $A_{1}$ denote the class of functions of the form

$$
f(z)=z+\sum_{k=2}^{\infty} a_{k} z^{k}
$$

which are analytic in the unit disc $U=\{z:|z|<1\}$, and let $S$ denote the subclass of $A_{1}$ consisting of analytic and univalent functions $f(z)$ in the unit disc $U$. We use $\Omega$ to denote the class of analytic functions $w(z)$ in $U$ satisfies the conditions $w(0)=0$ and $|w(z)| \leq|z|$ for $z \in U$.

Received September 3, 1993; revised September 26, 1994.

1991 Mathematics Subject Classification. 30C45.

Key words and phrases. Analytic, convex, modified Hadamard procuct, fractional calculus. 
For a function $f(z)$ in $S$, we define

$$
\begin{aligned}
& D^{0} f(z)=f(z), \\
& D^{1} f(z)=D f(z)=z f^{\prime}(z),
\end{aligned}
$$

and

$$
D^{n} f(z)=D\left(D^{n-1} f(z)\right) \quad(n \in \mathbb{N}=\{1,2, \ldots\}) .
$$

The differential operator $D^{n}$ was introduced by Salagean [7]. With the help of the differential operator $D^{n}$, we say that a function $f(z)$ belonging to $S$ is in the class $S(A, B, n, \alpha) \quad\left(-1 \leq A<B \leq 1,0<B \leq 1, n \in \mathbb{N}_{\mathcal{O}}=\mathbb{N} \cup\{0\}\right.$, and $\left.0 \leq \alpha<1\right)$ if and only if

$$
\frac{D^{n+1} f(z)}{D^{n} f(z)} \prec \frac{1+[B+(A-B)(1-\alpha)] z}{1+B z}, \quad z \in U .
$$

Equivalently, a function $f(z)$ of $S$ belongs to the class $S(A, B, n, \alpha)$ if and only if there exists a function $w(z) \in \Omega$ such that

$$
\frac{D^{n+1} f(z)}{D^{n} f(z)}=\frac{1+[B+(A-B)(1-\alpha)] w(z)}{1+B w(z)}, \quad z \in U
$$

It is easy to see that the condition (1.6) is equivalent to

$$
\left|\frac{\frac{D^{n+1} f(z)}{D^{n} f(z)}-1}{B \frac{D^{n+1} f(z)}{D^{n} f(z)}-[B+(A-B)(1-\alpha)]}\right|<1, \quad z \in U .
$$

Let $T$ denote the subclass of $S$ consisting of functions of the form

$$
f(z)=z-\sum_{k=2}^{\infty} a_{k} z^{k} \quad\left(a_{k} \geq 0\right) .
$$

Further, we define the class $T^{*}(A, B, n, \alpha)$ by

$$
T^{*}(A, B, n, \alpha)=S(A, B, n, \alpha) \cap T \text {. }
$$

We note that, by specializing the parameters $A, B, n$, and $\alpha$, we obtain the following subclasses studied by various authors:

(1) $T^{*}(-1,1, n, \alpha)=T(n, \alpha)($ Hur and Oh [4]);

(2) $T^{*}(-1,1,0, \alpha)=T^{*}(\alpha)$ and $T^{*}(-1,1,1, \alpha)=C(\alpha)$ (Silverman [9]);

(3) $T^{*}(-\beta, \beta, 0, \alpha)=S^{*}(\alpha, \beta)$ and $T^{*}(-\beta, \beta, 1, \alpha)=C^{*}(\alpha, \beta) \quad(0 \leq \alpha<1),(0<\beta \leq$

1) (Gupta and Jain [3]);

(4) $T^{*}(A, B, 0,0)=T_{1}^{*}(A, B)$ and $T^{*}(A, B, 1,0)=C_{1}(A, B)$. (Goel and Sohi [2]);

(5) $T^{*}(A, B, 0, \alpha)=T_{1}^{*}(A, B, \alpha)$ and $T^{*}(A, B, 1, \alpha)=C_{1}(A, B, \alpha)$ (Aouf [1]); 
(6) $T^{*}(-\beta, \mu \beta, 0, \alpha)=S^{*}(\alpha, \beta, \mu)$ and $T^{*}(-\beta, \mu \beta, 1, \alpha)=C^{*}(\alpha, \beta, \mu)(0 \leq \alpha<1),(0<$ $\beta \leq 1)$ and $(0 \leq \mu \leq 1)$ (Owa and Aouf [6]);

(7) $T^{*}(-\beta, \beta, n, \alpha)=S^{*}(\alpha, \beta, n)$, where $S^{*}(\alpha, \beta, n)$ represents the class of functions $f(z) \in T$ satisfying the condition

$$
\left|\frac{\frac{D^{n+1} f(z)}{D^{n} f(z)}-1}{\frac{D^{n+1} f(z)}{D^{n} f(z)}+1-2 \alpha}\right|<\beta, \quad z \in \in U
$$

where $0 \leq \alpha<1$, and $0<\beta \leq 1$.

\section{Coefficient Estimates}

Theorem 1. Let the function $f(z)$ be defined by (1.8). Then $f(z) \in$ $T^{*}(A, B, n, \alpha)\left(-1 \leq A<B \leq 1,0<B \leq 1, n \in \mathbb{N}_{0}\right.$, and $\left.0 \leq \alpha<1\right)$ if and only if

$$
\sum_{k=2}^{\infty} C_{k} a_{k} \leq(B-A)(1-\alpha)
$$

where

$$
C_{k}=k^{n}[(1+B)(k-1)+(B-A)(1-\alpha)] .
$$

The result is sharp.

Proof. Let $|z|=1$, then

$$
\begin{aligned}
& \left|D^{n+1} f(z)-D^{n} f(z)\right|-\left|B D^{n+1} f(z)-[B+(A-B)(1-\alpha)] D^{n} f(z)\right| \\
= & \left|-\sum_{k=2}^{\infty} k^{n}(k-1) a_{k} z^{k}\right|-\left|(B-A)(1-\alpha) z-\sum_{k=2}^{\infty} k^{n}[B(k-1)+(B-A)(1-\alpha)] a_{k} z^{k}\right| \\
\leq & \sum_{k=2}^{\infty} k^{n}[(1+B)(k-1)+(B-A)(1-\alpha)] a_{k}-(B-A)(1-\alpha) \leq 0 .
\end{aligned}
$$

Hence, by the principle of maximum modulus $f(z) \in T^{*}(A, B, n, \alpha)$.

Conversely, suppose that

$$
\left|\begin{array}{c}
\frac{\frac{D^{n+1} f(z)}{D^{n} f(z)}-1}{B \frac{D^{n+1} f(z)}{D^{n} f(z)}-[B+(A-B)(1-\alpha)]} \mid \\
-\sum_{k=2}^{\infty} k^{n}(k-1) a_{k} z^{k} \\
\frac{(B-A)(1-\alpha) z-\sum_{k=2}^{\infty} k^{n}[B(k-1)+(B-A)(1-\alpha)] a_{k} z^{k}}{(B-1) \quad z \in U .}
\end{array}\right|<1,
$$


Since $|\operatorname{Re}(z)| \leq|z|$ for all $z$, we have

$$
\operatorname{Re}\left\{\frac{\sum_{k=2}^{\infty} k^{n}(k-1) a_{k} z^{k}}{(B-A)(1-\alpha) z-\sum_{k=2}^{\infty} k^{n}[B(k-1)+(B-A)(1-\alpha)] a_{k} z^{k}}\right\}<1 .
$$

Choose values of $z$ on the real axis so that $\frac{D^{n+1} f(z)}{D^{n} f(z)}$ is real. Upon clearing the denominator in (2.3) and letting $z \longrightarrow 1^{-}$through real values, we obtain

$$
\sum_{k=2}^{\infty} k^{n}(k-1) a_{k} \leq(B-A)(1-\alpha)-\sum_{k=2}^{\infty} k^{n}[B(k-1)+(B-A)(1-\alpha)] a_{k}
$$

which implies that

$$
\sum_{k=2}^{\infty} k^{n}[(1+B)(k-1)+(B-A)(1-\alpha)] a_{k} \leq(B-A)(1-\alpha) .
$$

The result is sharp for the function

$$
f(z)=z-\frac{(B-A)(1-\alpha)}{C_{k}} z^{k} \quad(k \geq 2)
$$

Using Theorem 1, we have the following:

Corollary 1. Let the function $f(z)$ defined by (1.8) be in the class $T^{*}(A, B, n, \alpha)$. then we have

$$
a_{k} \leq \frac{(B-A)(1-\alpha)}{C_{k}} \quad(k \geq 2)
$$

The equality in (2.5) is attained for the function $f(z)$ given by (2.4).

Corollary 2. $T^{*}(A, B, n+1, \alpha) \subset T^{*}(A, B, n, \alpha)$ for $-1 \leq A<B \leq 1,0<B \leq$ $1, n \in \mathbb{N}_{0}$, and $0 \leq \alpha<1$.

\section{Distortion Theorems}

Theorem 2. Let the function $f(z)$ defined by (1.8) be in the class $T^{*}(A, B, n, \alpha)$. Then we have

$$
|z|-\frac{2^{i}(B-A)(1-\alpha)}{C_{2}}|z|^{2} \leq\left|D^{i} f(z)\right| \leq|z|+\frac{2^{i}(B-A)(1-\alpha)}{C_{2}}|z|^{2}
$$

for $z \in U$, where $0 \leq i \leq n$. the result is sharp. 
Proof. Note that $f(z) \in T^{*}(A, B, n, \alpha)$ if and only if $D^{i} f(z) \in T^{*}(A, B, n-i, \alpha)$, and that

$$
D^{i} f(z)=z-\sum_{k=2}^{\infty} k^{i} a_{k} z^{k}
$$

Using Theorem 1, we can get the result. Finally, we note that the equality in (3.1) is attained for the function $f(z)$ defined by

$$
D^{i} f(z)=z-\frac{2^{i}(B-A)(1-\alpha)}{C_{2}} z^{2} .
$$

This completes the proof of Theorem 2 .

Corollary 3. Let the function $f(z)$ defined by (1.8) be in the class $T^{*}(A, B, n, \alpha)$. Then we have

$$
|z|-\frac{(B-A)(1-\alpha)}{C_{2}}|z|^{2} \leq|f(z)| \leq|z|+\frac{(B-A)(1-\alpha)}{C_{2}}|z|^{2}
$$

for $z \in U$. The result is sharp for the function

$$
f(z)=z-\frac{(B-A)(1-\alpha)}{C_{2}} z^{2} .
$$

Proof. Taking $i=0$ in Theorem 2, we can easily show (3.8).

Corollary 4. Let the function $f(z)$ defined by (1.8) be in the class $T^{*}(A, B, n, \alpha)$. Then we have

$$
1-\frac{2(B-A)(1-\alpha)}{C_{2}}|z| \leq\left|f^{\prime}(z)\right| \leq 1+\frac{2(B-A)(1-\alpha)}{C_{2}}|z|
$$

for $z \in U$. The result is sharp for the function $f(z)$ given by (3.5).

Proof. Note that $D f(z)=z f^{\prime}(z)$. Hence, taking $i=1$ in Theorem 2, we have Corollary 4.

\section{Closure Theorems}

Let the functions $f_{\nu}(z) \quad(\nu=1,2)$ be defined by

$$
f_{\nu}(z)=z-\sum_{k=2}^{\infty} a_{k, \nu} z^{k} \quad\left(a_{k, \nu} \geq 0, \nu=1,2\right) .
$$

Employing the techniques used earlier by Silverman [9], Gupta and Jain [3], Hur and $\mathrm{Oh}[4]$ and Owa and Aouf [6], and with the aid of Theorem 1, we can prove the following: 
Theorem 3. The class $T^{*}(A, B, n, \alpha)$ is closed under convex linear combination.

As a consequence of Theorem 3 , there exists extreme points of the class $T^{*}(A, B, n, \alpha)$.

Theorem 4. Let $f_{1}(z)=z$ and

$$
f_{k}(z)=z-\frac{(B-A)(1-\alpha)}{C_{k}} z^{k} \quad(k \geq 2)
$$

for $-1 \leq A<B \leq 1,0<B \leq 1$, and $0 \leq \alpha<1$, Then $f(z)$ is in the class $T^{*}(A, B, n, \alpha)$ if and only if it can be expressed in the form

$$
f(z)=\sum_{k=1}^{\infty} \lambda_{k} f_{k}(z)
$$

where $\lambda_{k} \geq 0 \quad(k \geq 1)$ and $\sum_{k=1}^{\infty} \lambda_{k}=1$.

Corollary 5. The extreme points of the class $T^{*}(A, B, n, \alpha)$ are the functions $f_{k}(z)(k \geq 1)$ given by Theorem 4 .

\section{Modified Hadamard Products}

Let the functions $f_{\nu}(z)(\nu=1,2)$ be defined by (4.1). The modified Hadamard product of $f_{1}(z)$ and $f_{2}(z)$ is defined by

$$
f_{1} * f_{2}(z)=z-\sum_{k=2}^{\infty} a_{k, 1} a_{k, 2} z^{k} .
$$

Employing the technique used earlier by Schild and Silverman [8], we can prove the following:

Theorem 5. Let the functions $f_{\nu}(z)(\nu=1,2)$ defined by $(4,1)$ be in the class $T^{*}(A, B, n, \alpha)$. Then we have

$$
f_{1} * f_{2}(z) \in T^{*}(A, B, n, \beta(A, B, n, \alpha))
$$

where

$$
\beta(A, B, n, \alpha)=\frac{2^{n}-(B-A)(2 B+1-A)\left[\frac{2^{n}(1-\alpha)}{C_{2}}\right]^{2}}{2^{n}-\left[\frac{2^{n}(B-A)(1-\alpha)}{C_{2}}\right]^{2}} .
$$

The result is sharp for the functions

$$
f_{\nu}(z)=z-\frac{(B-A)(1-\alpha)}{C_{2}} z^{2} \quad(\nu=1,2) .
$$


Theorem 6. Let the functions $f_{\nu}(z) \quad(\nu=1,2)$ defined by (4.1) be in the class $T^{*}(A, B, n, \alpha)$. Then the function

$$
h(z)=z-\sum_{k=2}^{\infty}\left[a_{k, 1}^{2}+a_{k, 2}^{2}\right] z^{k}
$$

belongs to the class $T^{*}(A, B, n, \gamma(A, B, n, \alpha))$, where

$$
\gamma(A, B, n, \alpha)=\frac{2^{n}-2(B-A)(2 B+1-A)\left[\frac{2^{n}(1-\alpha)}{C_{2}}\right]^{2}}{2^{n}-2\left[\frac{2^{n}(B-A)(1-\alpha)}{C_{2}}\right]^{2}} .
$$

The result is sharp for the functions $f_{\nu}(z)(\nu=1,2)$ defined by (5.4).

\section{Radii of Close-to-Convexity, Starlikeness and Convexity}

By using Theorem 1, we can prove the following:

Theorem 7. Let the function $f(z)$ defined by (1.8) be in the class $T^{*}(A, B, n, \alpha)$, then $f(z)$ is close-to-convex of order $\rho(0 \leq \rho<1)$ in $|z|<R_{1}$, where

$$
R_{1}=\inf _{k}\left[\frac{(1-\rho) C_{k}}{k(B-A)(1-\alpha)}\right]^{\frac{1}{k-1}} \quad(k \geq 2) .
$$

The result is sharp, with the extremal function $f(z)$ given by (2.4).

Theorem 8. Let the function $f(z)$ defined by (1.8) be in the class $T^{*}(A, B, n, \alpha)$, then $f(z)$ is starlike of order $\rho(0 \leq \rho<1)$ in $|z|<R_{2}$, where

$$
R_{2}=\inf _{k}\left[\frac{(1-\rho) C_{k}}{(k-\rho)(B-A)(1-\alpha)}\right]^{\frac{1}{k-1}} \quad(k \geq 2) .
$$

The result is sharp, with the extremal function $f(z)$ given by (2.4).

Corollary 6. Let the function $f(z)$ defined by (1.8) be in the class $T^{*}(A, B, n, \alpha)$, then $f(z)$ is convex of order $\rho(0 \leq \rho<1)$ in $|z|<R_{3}$, where

$$
R_{3}=\inf _{k}\left[\frac{(1-\rho) C_{k}}{k(k-\rho)(B-A)(1-\alpha)}\right]^{\frac{1}{k-1}} \quad(k \geq 2) .
$$

The result is sharp, with the extremal function $f(z)$ given by (2.4). 


\section{Integral Operators}

Theorem 9. Let the function $f(z)$ defined by (1.8) be in the class $T^{*}(A, B, n, \alpha)$, and let $c$ be a real number such that $c>-1$. Then the function $F(z)$ defined by

$$
F(z)=\frac{c+1}{z^{c}} \int_{0}^{z} t^{c-1} f(t) d t
$$

also belongs to the class $T^{*}(A, B, n, \alpha)$.

Proof. From the representation of $F(z)$, it follows that

$$
F(z)=z-\sum_{k=2}^{\infty} b_{k} z^{k}
$$

where

$$
b_{k}=\left(\frac{c+1}{c+k}\right) a_{k}
$$

Therefore,

$$
\sum_{k=2}^{\infty} C_{k} b_{k}=\sum_{k=2}^{\infty} C_{k}\left(\frac{c+1}{c+k}\right) a_{k} \leq \sum_{k=2}^{\infty} C_{k} a_{k} \leq(B-A)(1-\alpha),
$$

since $f(z) \in T^{*}(A, B, n, \alpha)$. Hence, by Theorem $1, F(z) \in T^{*}(A, B, n, \alpha)$.

Finally by using Theorem 1 , we can prove the following theorem:

Theorem 10. Let $c$ be a real number such that $c>-1$. If $F(z) \in$ $T^{*}(A, B, n, \alpha)$, then the function $f(z)$ defined by (7.1) is univalent in $|z|<R^{*}$, where

$$
R^{*}=\inf _{k}\left[\frac{(c+1) C_{k}}{k(c+k)(B-A)(1-\alpha)}\right]^{\frac{1}{k-1}} \quad(k \geq 2) .
$$

The result is sharp for the function

$$
f(z)=z-\frac{(c+k)(B-A)(1-\alpha)}{(c+1) C_{k}} z^{k} .
$$

\section{Fractional Calculus}

We begin with the statements of the following definitions of fractional calculus (that is, fractional derivatives and fractional integrals) which were defined by Owa [5] and used recently by Srivastava and Owa [10].

Definition 1. The fractional integral of order $\lambda$ is defined, for a function $f(z)$, by

$$
D_{z}^{-\lambda} f(z)=\frac{1}{\Gamma(\lambda)} \int_{0}^{z} \frac{f(\zeta)}{(z-\zeta)^{1-\lambda}} d \zeta \quad(\lambda>0)
$$


where $f(z)$ is an analytic function in a simply-connected region of the $z$-plane containing the origin, and the multiplicity of $(z-\zeta)^{\lambda-1}$ is removed by requiring $\log (z-\zeta)$ to be real when $z-\zeta>0$.

Definition 2. The fractional derivative of order $\lambda$ is defined, for a function $f(z)$, by

$$
D_{z}^{\lambda} f(z)=\frac{1}{\Gamma(1-\lambda)} \frac{d}{d z} \int_{0}^{z} \frac{f(\zeta)}{(z-\zeta)^{\lambda}} d \zeta \quad(0 \leq \lambda<1),
$$

where $f(z)$ is constrained, and the multiplicity of $(z-\zeta)^{-\lambda}$ is removed, as in Definition 1.

Definition 3. Under the hypotheses of Definition 2, the fractional derivative of order $n+\lambda$ is defined, for a function $f(z)$, by

$$
D_{z}^{n+\lambda} f(z)=\frac{d^{n}}{d z^{n}} D_{z}^{\lambda} f(z) \quad\left(0 \leq \lambda<1 ; n \in \mathbb{N}_{0}\right)
$$

Employing the technique used earlier by Srivastava and Owa [10], we can prove the following:

Theorem 11. Let the function $f(z)$ defined by (1.8) be in the class $T^{*}(A, B, n, \alpha)$. Then we have

$$
\left|D_{z}^{-\lambda}\left(D^{i} f(z)\right)\right| \geq \frac{|z|^{1+\lambda}}{\Gamma(2+\lambda)}\left\{1-\frac{2^{i+1}(B-A)(1-\alpha)}{(2+\lambda) C_{2}}|z|\right\}
$$

and

$$
\left|D_{z}^{-\lambda}\left(D^{i} f(z)\right)\right| \leq \frac{|z|^{1+\lambda}}{\Gamma(2+\lambda)}\left\{1+\frac{2^{i+1}(B-A)(1-\alpha)}{(2+\lambda) C_{2}}|z|\right\}
$$

for $\lambda>0,0 \leq i \leq n$, and $z \in U$. The result is sharp for the function

$$
D_{z}^{-\lambda}\left(D^{i} f(z)\right)=\frac{z^{1+\lambda}}{\Gamma(2+\lambda)}\left\{1-\frac{2^{i+1}(B-A)(1-\alpha)}{(2+\lambda) C_{2}} z\right\}
$$

or

$$
\left(D^{i} f(z)\right)=z-\frac{2^{i}(B-A)(1-\alpha)}{C_{2}} z^{2} .
$$

Theorem 12. Let the function $f(z)$ defined by (1.8) be in the class $T^{*}(A, B, n, \alpha)$. Then we have

$$
\left|D_{z}^{\lambda}\left(D^{i} f(z)\right)\right| \geq \frac{|z|^{1-\lambda}}{\Gamma(2-\lambda)}\left\{1-\frac{2^{i+1}(B-A)(1-\alpha)}{(2-\lambda) C_{2}}|z|\right\}
$$

and

$$
\left|D_{z}^{\lambda}\left(D^{i} f(z)\right)\right| \leq \frac{|z|^{1-\lambda}}{\Gamma(2-\lambda)}\left\{1+\frac{2^{i+1}(B-A)(1-\alpha)}{(2-\lambda) C_{2}}|z|\right\}
$$


for $0 \leq \lambda<1,0 \leq i \leq n-1$, and $z \in U$. The result is sharp for the function $f(z)$ given by

$$
D_{z}^{\lambda}\left(D^{i} f(z)\right)=\frac{z^{1-\lambda}}{\Gamma(2-\lambda)}\left\{1-\frac{2^{i+1}(B-A)(1-\alpha)}{(2-\lambda) C_{2}} z\right\}
$$

or by $D^{i} f(z)$ given by $(8.7)$.

\section{Fractional Integral Operator}

We need the following definition of fractional integral operator given by Srivastava, Saigo and Owa [11].

Definition 4. For real numbers $\beta>0, \gamma$ and $\delta$, the fractional integral operator $I_{o, z}^{\beta, \gamma, \delta}$ is defined by

$$
I_{o, z}^{\beta, \gamma, \delta} f(z)=\frac{z^{-\beta-\gamma}}{\Gamma(\beta)} \int_{0}^{z}(z-t)^{\beta-1} F\left(\beta+\gamma,-\delta ; \beta ; 1-\frac{t}{z}\right) f(t) d t
$$

where $f(z)$ is an analytic function in a simply-connected region of the z-plane containing the origin with the order

$$
f(z)=O\left(|z|^{\varepsilon}\right), \quad z \longrightarrow 0
$$

where

$$
\begin{gathered}
\varepsilon>\max (0, \gamma-\delta)-1, \\
F(a, b ; c ; z)=\sum_{k=0}^{\infty} \frac{(a)_{k}(b)_{k}}{(c)_{k}(1)_{k}} z^{k}
\end{gathered}
$$

where $(\nu)_{k}$ is the Pochhammer symbol defined by

$$
(\nu)_{k}=\frac{\Gamma(\nu+k)}{\Gamma(\nu)}= \begin{cases}1 & (k=0) \\ \nu(\nu+1) \cdots(\nu+k-1) & (k \in \mathbb{N})\end{cases}
$$

and the multiplicity of $(z-t)^{\beta-1}$ is removed by requiring $\log (z-t)$ to be real when $z-t>0$.

Remark. For $\gamma=-\beta$, we note that

$$
I_{o, z}^{\beta,-\beta, \delta} f(z)=D_{z}^{-\beta} f(z) .
$$

In order to prove our results for the fractional integral operator, we have to recall here the following lemma due to Srivastava, Saigo and Owa [11].

Lemma1. If $\beta>0$ and $k>\gamma-\delta-1$, then

$$
I_{o, z}^{\beta, \gamma, \delta} z^{k}=\frac{\Gamma(k+1) \Gamma(k-\gamma+\delta+1)}{\Gamma(k-\gamma+1) \Gamma(k+\beta+\delta+1)} z^{k-\gamma} .
$$


Employing the technique used earlier by Srivastive, Saigo and Owa [11] and with the aid of the above lemma, we can prove the following:

Theorem 13. Let $\beta>0, \gamma<2, \beta+\delta>-2, \gamma-\delta<2, \gamma(\beta+\delta) \leq 3 \beta$. If the function $f(z)$ defined by $(1.8)$ is in the class $T^{*}(A, B, n, \alpha)$, then

$$
\left|I_{o, z}^{\beta, \gamma, \delta} f(z)\right| \geq \frac{\Gamma(2-\gamma+\delta)|z|^{1-\gamma}}{\Gamma(2-\gamma) \Gamma(2+\beta+\delta)}\left\{1-\frac{2(B-A)(1-\alpha)(2-\gamma+\delta)}{(2-\gamma)(2+\beta+\delta) C_{2}}|z|\right\}
$$

and

$$
\left|I_{o, z}^{\beta, \gamma, \delta} f(z)\right| \leq \frac{\Gamma(2-\gamma+\delta)|z|^{1-\gamma}}{\Gamma(2-\gamma) \Gamma(2+\beta+\delta)}\left\{1+\frac{2(B-A)(1-\alpha)(2-\gamma+\delta)}{(2-\gamma)(2+\beta+\delta) C_{2}}|z|\right\}
$$

for $z \in U$, where

$$
U_{o}= \begin{cases}U & (\gamma \leq 1) \\ U-\{0\} & (\gamma>1)\end{cases}
$$

The equalities in (9.5) and (9.6) are attained for the function $f(z)$ given by (3.5).

\section{Acknowledgements}

The authors would like to thank the referee of the paper for his helpfull suggestions.

\section{References}

[1] M. K. Aouf, "A generalization of multivalent functions with negative coefficients," J. Korean Math. Soc., 25(1988), no. 1, 53-66.

[2] R. M. Goel and N. S. Sohi, "Multivalent functions with negative coefficients," Indian J. Pure Appl. Math., 12 (1981), no. 7, 844-853.

[3] V. P. Gupta and P. K. Jain, "Certain classes of univalent functions with negative coefficients," Bull. Austral. Math. Soc., 14 (1976), 409-416.

[4] M. D. Hur and G. M. Oh, "On Certain class of analytic functions with negative coefficients," Pusan Kyongnam Math. J., 5 (1989), 69-80.

[5] S. Owa, "On the distortion theorems," I, Kyungpook Math. J., 18 (1978), 53-59.

[6] S. Owa and M. K. Aouf, "On subclasses of univalent functins with negative coefficients," Pusan Kyongnam Math. J., 4 (1988), 57-73.

[7] G. S. Salagean, "Subclasses of univalent functions," Lecture.notes in Math.(Springer-Verlag), 1013 (1983), 362-372.

[8] A. Schild and H. Silverman, "Convolutions of univalent functions with negative coefficients," Ann. Univ. Marice Curie-Sklodowska Sect. A, 29 (1975), 99-107.

[9] H. Silverman, "Univalent functions with negative coefficients," Proc. Amer Math. Soc., 51 (1975),

109-116.
[10] H. M. Srivastava and S. Owa, "An application of the fractional derivative," Math. Japon., 29 (1984), 383-389.

[11] H. M. Srivastava, M. Saigo and S. Owa, "A class of distortion theorems involving certain operators of fractional calculus," J. Math. Anal. Appl., 131 (1988), 412-420.

Department of Mathematics, Faculty of Science, University of Mansoura, Mansoura, EGYPT. 